\title{
Erratum to: Synthesis and antimicrobial activity of tetrazolo[1,5-a]quinoline-4-carbonitrile derivatives
}

\author{
Amol H. Kategaonkar • Vilas B. Labade • \\ Pravin V. Shinde $\cdot$ Atul H. Kategaonkar • \\ Bapurao B. Shingate $\cdot$ Murlidhar S. Shingare
}

Published online: 1 July 2010

(C) Springer-Verlag 2010

\section{Erratum to: Monatsh Chem}

\section{DOI 10.1007/s00706-010-0324-2}

Unfortunately, in Scheme 1 part of the legend was missing.

The correct scheme is given here:

Scheme 1<smiles>[R]c1cc2cc(C=O)c(Cl)nc2c([R])c1[R7]</smiles>

Reagents and conditions: (a) Sodium azide, $\mathrm{DMSO} / \mathrm{AcOH}$, rt, 4-5 h;

(b) Hydroxyl amine hydrochloride/formic acid, reflux, $3 \mathrm{~h}$<smiles>[R]c1cc2cc(C=O)c3nnnn3c2c([R])c1[R]</smiles>

2a-2i<smiles>[R]c1cc2cc(C#N)c3nnnn3c2c([R])c1[R]</smiles>

3a-3i
a) $R^{1}=R^{2}=R^{3}=H$;
c) $R^{1}=R^{3}=H ; R^{2}=M e ;$ d) $R^{1}=R^{2}=H ; R^{3}=M e$;
b) $R^{2}=R^{3}=H ; R^{1}=M e$;
e) $R^{2}=R^{3}=H ; R^{1}=O M e ;$ f) $R^{1}=R^{3}=H ; R^{2}=O M e$;
g) $R^{1}=R^{2}=H ; R^{3}=$ OMe; h) $R^{2}=R^{3}=H ; R^{1}=$ OEt;
i) $R^{1}=R^{2}=H ; R^{3}=E t$.

The online version of the original article can be found under doi:10.1007/s00706-010-0324-2.

\footnotetext{
A. H. Kategaonkar · V. B. Labade · P. V. Shinde ·

B. B. Shingate $\cdot$ M. S. Shingare $(\square)$

Department of Chemistry, Dr. Babasaheb Ambedkar

Marathwada University, Aurangabad,

Maharashtra 431 004, India

e-mail: prof_msshingare@ rediffmail.com

A. H. Kategaonkar
Pharmacology Laboratory, Maharashtra Institute of Pharmacy,
Pune, Maharashtra 411 038, India
} 